\title{
Evaluation of occupational exposure to waste anaesthetic gases in the inflammatory and oxidative stress biomarkers in medical residents
}

\author{
Mariana G. Braz*, Lorena M.C. Lucio, Ludimila O. Resende, Daniel A.A. Filho, José R.C. \\ Braz JR, Leandro G. Braz \\ Botucatu Medical School, UNESP - Sao Paulo State University, Botucatu, SP, Brazil
}

\section{BACKGROUND AND GOAL}

Occupational exposure to anaesthetics is thought to exert adverse effects in operating room personnel. It is known that inflammation and oxidative stress are linked processes; however, it lacks in the literature reports about the effects of occupational exposure to anaesthetics in the inflammationoxidative stress pathways. Thus, this study aimed to evaluate inflammatory cytokines and oxidative stress in medical residents occupationally exposed to waste anaesthetic gases.

\section{METHODS}

The Ethical Committee of the Institution (Botucatu Medical School - University Hospital) approved the protocol of the study, which included 30 physicians, at the end of their residency program (three years), and who were allocated into two groups:

* exposed group $(\mathbf{n}=15)$ : anaesthesiology and surgery areas exposed to waste anaesthetic gases (isoflurane, sevoflurane and nitrous oxide)

* control group $(\mathbf{n}=15)$ : medical residents not exposed to waste anaesthetic gases (internal medicine)

- exclusion criteria

Blood samples were collected at the same time for both groups, and plasma aliquots were used for evaluation of:

- pro-inflammatory interleukins (IL-6, IL-8 and IL-17A)

- oxidative stress (lipid peroxidation: malondialdehyde-MDA and 4-hydroxynonenal-4HNE, and the antioxidative status using the total antioxidant performance-TAP)
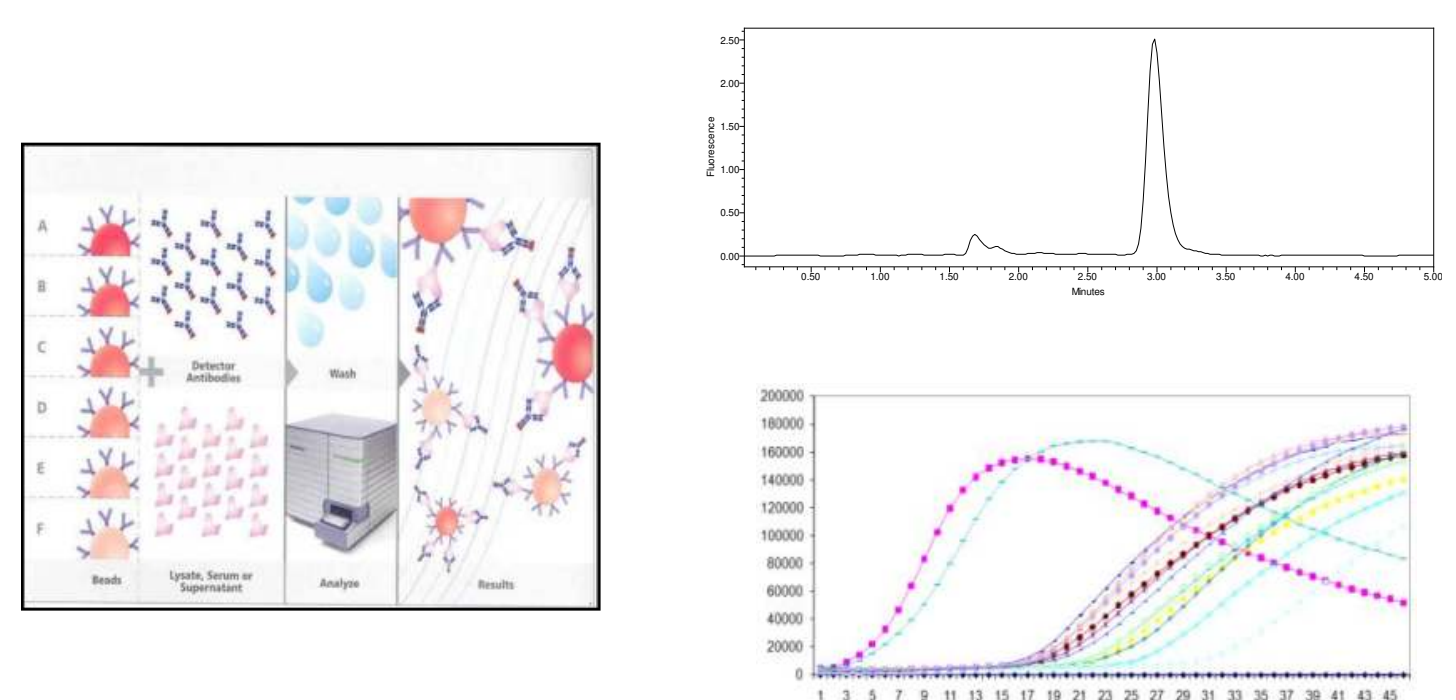

\section{RESULTS}

Table 1- Demographic data in both groups evaluated $(X \pm S D$ or absolute number)

\begin{tabular}{ccc}
\hline Characteristics & Control group & Exposed group \\
\hline Age (years) & $27.8 \pm 1.3$ & $28.2 \pm 1.0$ \\
Sex (M/F) & $9 / 6$ & $10 / 5$ \\
BMI $\left(\mathbf{k g} / \mathbf{m}^{2}\right)$ & $23.6 \pm 1.7$ & $24.0 \pm 1.6$ \\
\hline $\mathrm{p}>0.05$ & &
\end{tabular}

Table 2- Plasma inflammatory cytokines evaluated in both groups $(\mathrm{X} \pm \mathrm{SD})$

\begin{tabular}{ccc}
\hline Cytokines $(\mathrm{pg} / \mathrm{mL})$ & Control group & Exposed group \\
\hline IL-6 & $3.6 \pm 1.2$ & $4.1 \pm 1.3$ \\
IL-8 & $5.5 \pm 1.5$ & $9.7 \pm 1.4^{*}$ \\
IL-17A & $9.6 \pm 3.2$ & $19.8 \pm 3.8^{*}$ \\
\hline
\end{tabular}

$* \mathbf{p}<0.05$

Table 3- Oxidative stress biomarkers evaluated in plasma samples from both groups $(\mathrm{X} \pm \mathrm{SD})$

\begin{tabular}{lcc}
\multicolumn{1}{c}{ Parameters } & Control group & Exposed group \\
\hline MDA $(\boldsymbol{\mu M})$ & $0.5 \pm 0.2$ & $0.5 \pm 0.1$ \\
$\mathbf{4 - H N E}(\boldsymbol{\mu g} / \mathbf{m L})$ & $8.8 \pm 5.1$ & $9.8 \pm 5.7$ \\
$\mathbf{T A P}(\%)$ & $77.3 \pm 6.0$ & $78.8 \pm 7.9$ \\
\hline $\mathrm{p}>0.05$ & \\
& \\
& CONCLUSION
\end{tabular}

The findings suggest, for the first time, an inflammatory response without alterations in the antioxidant status in medical residents exposed for three years to waste anaesthetic gases. 